\title{
Research on Laboratory Teaching in Fine Arts Colleges
}

\author{
Yue Wang \\ Art \& Design School, Wuhan University of Science and Technology
}

Keywords: Laboratory opening; Fine arts university; Laboratory equipments

\begin{abstract}
Laboratory opening is an essential procedure for the reformation and development of general fine arts university laboratories in our country, and is also an important aspect for improving the school level. Laboratory opening improves not only the reformation of laboratory teaching and abilities of students' experiences but also the usage efficiency of the laboratory equipments. By incorporating the actual situation of our fine arts university, the way which the opening of time and teaching contents simultaneously is adopted. To manage the laboratory using the opening laboratory management system based on campus network, both the experimental abilities and innovative spirit of students are developed efficiently.
\end{abstract}

\section{Introduction}

Opening of Laboratory is a long topic.It has become indispensable to develop innovative means of talent.The experimental teaching experiment in improving the utilization of resources to stimulate the enthusiasm of students, to develop students ability to work independently and innovative thinking has made a great success, so it is widely used all colleges and universities. In particular, the Ministry of Education Undergraduate teaching evaluation, the school's basic course lab conditions have improved to varying degrees, making it possible to open laboratory.Open lab includes three meanings: One is the laboratory to open in time to implement full use of existing conditions, as much as possible for students to create convenient conditions and good service, no time limit so that students can complete the experimental tasks at any time in the lab. The second is an open experiment content, to provide students with many services as possible to enable students to conduct comprehensive and creative design experiment to enhance the creative ability of students. Third, to strengthen laboratory management, to establish sound management and protection system to ensure that students be introduced into the laboratory.

\section{Open lab experiment time, to ensure that students enter the lab at any time}

Laboratory experiments will undoubtedly increase the time and the experimental laboratory managers guide the workload of teachers, if only one or a few cases, the students open and experimental laboratory guidance, laboratory managers and lead Experiment great waste of time instructor, to address this problem, the general practice is to develop an open school schedule, students can within the time specified in the schedule to the laboratory experiments, the general provisions of this schedule can accommodate the time The number of students no more than the number of students should participate in the much larger, although students can be flexible in the time allocation within their own time, but the students choose to experiment a lot of time limitations and fail to open lab time real effect. The way we use the students make an appointment, when there are enough students choose to experiment with a time period when students can experiment, so that is open to the laboratory to ensure that the experimental time, while maximizing the conservation laboratory management and guidance to staff workload experiments. Experimental guide to Basic Computer, for example, the students also need to experiment on 3,000 people, 60 of each laboratory capacity, and if the experiment can be set at least 50 the number of people say, according to the worst case, need to be test managers and test to increase the workload of teachers for the guidance of the total workload of $16.67 \%$, under normal circumstances, the increased workload will not exceed $10 \%$ 
of the total workload. As for the lack of time a few students choose to focus not reach the required minimum number of test requirements, inform students to re-select the experimental time.

Before the emergence of open technology, the operating system running is on top of the hardware, the application process can acquire and obtain the right to use hardware by means of interruption, these hardware can be including controllers, memory, hard drives and food network laboratory card and so on. The dispatching work of hardware cab be dealt by the operating system, when multiple servers provide the service together, some of servers may be in the idle situation, while others are in busy situation, but the utilization rate can even be reached. This unbalanced usage of resources and uneven situation can be encountered by traditional physical machine easily, which is also relatively difficult to be solved.

After the emergence of open technology, there is a change in the physical machine model, which can be shown in Fig. 1. The host operating system is no longer in charge of management hardware, but it adds a layer in the middle of the virtual machine monitor, which is responsible for scheduling the use of the hardware. The disadvantage of this model is able to overcome the above-mentioned conventional physical machine so that the hardware utilization can be maximized and improved .The open technology is the resource of computer's hardware, such as memory, food network laboratory, and storage that are assigned with abstract meaning and converted to the client operating system that can enable the client operating system adjust these resources better than the traditional way. These virtual resources are not restricted by the current architecture, geography or physical state of the hardware resource. It can expand the capacity of the hardware, moreover, the software re-configuration process can be simplified. Open can be used to simulate multiple concurrent single stimulation, allowing a platform to exist a number of different operating systems at the same time, and the application program can run independently within their respective spaces without affecting each other, thus it can increase the utilization and efficiency of the physical machine significantly.

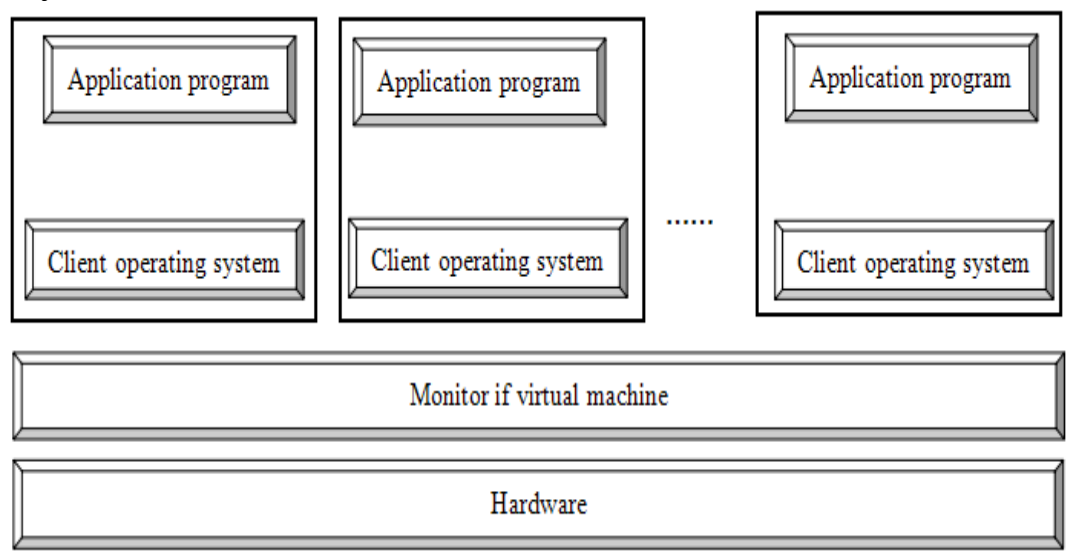

Fig.1 The Architecture open System

\section{The contents of the reform experiment, the innovative capacity of students}

Open Laboratory of Education to meet the requirements of first consideration in the arrangement of teaching students the training objectives, laws of cognition and learning characteristics, the past emphasis on imparting knowledge and theory, and experimental teaching only as a means to verify the theory. After the teaching experiment to implement an open, experimental teaching not only complement and reinforce classroom knowledge, students more integrated use of classroom knowledge to solve practical problems means. Therefore, students should first do a good job in opening up the basis of laboratory experiments, learn the basic knowledge, and then carrying out comprehensive training to achieve the purpose with ease. To solve these problems, we optimized experimental teaching content.

3.1 Construction of three-dimensional experimental teaching system

In order to meet the needs of students at different levels, we designed a three-dimensional teaching system, in this system, including the basic experiment, a comprehensive experimental and open 
experiment, the basic experiment is that each student must complete the experiment content, these experiments large part is the verification of classroom knowledge, to deepen and consolidate the students learned the purpose of the classroom; comprehensive experiment requires most students to complete, is the integrated use of classroom knowledge, ability; open experiment requires a small part of the school has spare capacity of the students, mainly students ability to innovate.

\subsection{Selected basic experiment}

Featured content knowledge in the learning of each point, all the contents of the corresponding experimental design, and in each experiment, are left thinking questions require students to seriously consider pop star. Experiments to the student, teacher effectiveness through student experiments and submit the job of teaching this stage of examination results, to enable students to complete hands-on, the ability of mental and physical training to enhance the overall quality of the training.

3.3 Undertake a comprehensive training program

This stage is to train students primarily the ability of the integrated use of knowledge, their culture is not the object of all students studying this course, but the school does have spare capacity of students so these students form groups of 3-4, select the title, the required time to complete an entire project. Specific projects such as the basic course in computer software requires students to design a "personnel management system", "student achievement management system", 'library management system "in the project during the training period to complete the project through the training phase to consolidate the basis of the results skilled engineering problem solving purposes.

Training is very important to the design of the project, whose main principle is to enable students to have an entry point to the various chapters of the experiment also the content and point of contact between the training programs, but also to eventually form a work. At this stage, teachers to "mentor" status appears to guide the students to participate in training programs for students to answer questions and check the completion of student projects, and timely for the students to summarize, sum up programming skills and grammar. Which guide the students involved in the project is not simply assign a task, but through a number of related procedures to enable students to hands-on exercise to achieve, can not make students feel helpless. Tour guide students through the completion of observations, ask students to submit the relevant documents and related works to test the wooden stage of education quality.

3.4 Open additional experiments to strengthen the innovation ability of students

Open experiment that students self-designed experiments. The content of student self-design experiments, test steps, test equipment and test instruments required to achieve the expected results, after review by teachers, students to the laboratory experiments. Experimental teaching initiative is to give students adequate to provide students with easy conditions and the environment, open throughout the course of the experiment learned to solve their own problems, analysis of experimental phenomena appear, and the evaluation results correctness, so that they can defeat the mind, a successful experiment in access to scientific quality and innovation.

\subsection{To carry out innovative experimental projects}

Students to actively explore innovative experiment as the main reforms to stimulate students to think creatively and innovation, driven by the students at the fine arts university level training in scientific research and invention, to enhance our school practice teaching, practical ability to improve student, school launched in 2011 on undergraduate learning and innovative experimental research project, called for scientific research and invention have a strong interest in majoring in science courses have spare capacity and good grades, I graduated from grade school full-time undergraduate students of non-declare, the approved supervisor with the help of students in independent study learning, self-designed experimental methods, organization of equipment and materials, implementation of the experiment, analysis and processing data, writing lab reports and so on. The student declaration on the nature and the amount of funding needed to carry out the project, the school to put into each project, ranging from project funding. 


\section{Using scientific means to strengthen the management of open laboratory}

Good system must have a scientific management methods as a support for managing the teaching of open laboratory, we developed an open campus -based laboratory management system, in this system, the lab announced the first open test of the subject, can be Open laboratory for experiments and laboratory capacity, students choose a good time to experiment, laboratory experiment in online booking, waiting for laboratory management approval, students approved to participate in time to the laboratory experiments, students also provide an experimental system assessment list, after the end of the experiment, experimental guide teachers in the system records the student's achievement. Meanwhile, the system also provides an open declaration of the experiment and management functions.

Campus-based experiment management system open to use by persons classified as administrators, teachers and students of three functional modules, each perform different functions.

4.1 administrators use the system to complete the function

Administrator posted on a Web page with the main laboratory, lab-related announcements, laboratory presentation, laboratory personnel, teachers, the basic situation, the management of laboratory equipment, etc., the most important feature is experimental curriculum, system management Members set up by the laboratory of experimental courses and teaching programs should be created for each course of the pilot project, only the set of pilot projects, the experimental teachers can arrange the experiment time, so students can choose.

4.2 The teachers use the system to complete the test function

Teachers to complete arrangements for the main experiment each experiment the opening hours for students, students in accordance with the scheduled time for teachers, student teachers after booking the reservation information for approval, prior to each experiment, the students front of the card in the laboratory to confirm status before entering the laboratory experiments, teachers can also be managed on student achievement, student performance is divided into prep results, experimental results, experimental results and other reports. Another function of the teachers reported the students set up an experiment since the approval of the project, and declare the project the students made revisions.

\section{The effectiveness of open laboratory}

Laboratory of the implementation of the basic experiment to break the traditional experimental teaching content in accordance with the provisions stipulated time to complete the mandatory requirements, students are free to mix their own arrangements of time and content of the experiment, the students truly become the protagonist of course, the teacher consultants for students change. Students to design a certain innovative pilot projects, teachers, students experiment for problems or ideas put forward guidance, the students formed their own process of doing a deep understanding of professional knowledge and insights. Innovation ability of students and innovation has been greatly improved.

Computer lab open since 2004, the cumulative increase in opening hours over 5000 hours, the students or teachers to be provided from a comprehensive training program for students close to more than 1,200 . Train the students by opening up the computer application ability, a large number of students through the National Computer Rank Examination, Microsoft certification exams, a group of students for many more social enterprises to develop a variety of management systems, websites and other applications, a more extensive treatment Chu Social Science students for the school examination system was developed, held in January 2010 has been applied to the final exam, students take the exam more than 3,000.

Electrical and Electronic Laboratory emphasis on the ability of electronic design students, foreign students in schools in recent years received a number of various types of electronic contest awards, only the 2009 e-contest to get Grand Prize of Liaoning Province 3, 12 first prize, second Prize 6 and third 12 good results. 
Mechanical Principles of Machine Design Laboratory focus on the students ability in recent years, access to students in schools outside the competition awards a number of various types of machinery, Liaoning Province in 2008 to get 3 mechanical Competition first prize, second prize of 3, third place 6, 2009 to receive the second prize of Liaoning Province, 6 mechanical competition, third place 6 of the good results.

\section{Conclusion}

Open experimental teaching students to solve the traditional experiments and pilot projects experimental time constraints, the experiments focused on the ability of students, in the course of the experiment, the students from passive to active real, in promoting the theory and practice with the same time, develop the students ability to innovate. Students to experiment by opening the vision to expand and deepen the understanding of knowledge. Meanwhile, the open Experiment increase the utilization of teaching equipment, without additional equipment investment and investment in the case of experimental space, increasing the number of students into the lab and time in cultivating applied talents played an important role.

\section{References}

[1] Zhou Xi-liang Reforming the Teaching Pattern of the Course of Experiment Enhancing the Students’ Creatility[J]. Journal of Xingtai Vocational and Technical, 2006(23),3:16-17.

[2] Xin Li ,Linna Xu Computer software foundation practice teaching research[J] Journal of Liaoning fine arts university of Technology , 2010,( 01), 118-120.

[3] ZHOU Gang The Researches and Practices on Laboratory Opening[J].Journal of Tongren fine arts university,2008.(4).78-79.

[4] Guo Wei qiang; Du Zhi qiang Exploration of the Opening Model for Experimental Teaching [J]. Research and Exploration in Laboratory.2003.(5).7-8.

[5] Agarwal N, Liu H, Tang L,2012.Modeling blogger influence in a community, Social food network laboratory Analysis and Mining,Vol.2, pp.139-162,February.

[6] Neiger, Gil, A. Santoni, F. Leung, D. Rodgers, R. Uhlig. 2000. Intel Virtualization Technology: Hardware Support for Efficient Processor Virtualization. Intel Technology Journal, vol. 103, pp:167-178.

[7] Samuel T.K, George W. Dunlap and Peter M. C hen. 2005. Debugging operating systems with time-traveling virtual machines. USENIX Annual Technical Conference, General Track, pp:1-15. 